\title{
Pinning adaptive synchronization analysis of linearly coupled delayed RDNNs with unknown time-varying coupling strengths
}

\author{
Weiyuan Zhang ${ }^{1 *}$, Junmin $\mathrm{Li}^{2}$ and Minglai Chen ${ }^{2}$
}

\author{
Correspondence: ahzwy@163.com \\ ${ }^{1}$ Institute of Mathematics and \\ Applied Mathematics, Xianyang \\ Normal University, Xianyang, \\ 712000, P.R. China \\ Full list of author information is \\ available at the end of the article
}

\begin{abstract}
This paper investigates the pinning synchronization analysis of linearly coupled reaction-diffusion neural networks (RDNNs) with unknown time-varying coupling strengths and unknown time-varying delay. By constructing a Lyapunov-Krasovskii-like composite energy functional (CEF) and applying the well-known LaSalle's invariance principle, an adaptive learning control is designed to guarantee the asymptotic convergence of the synchronization error, several sufficient conditions of the synchronization are derived. Compared with the existing results, the update laws do not need the information of the characteristics of the identical node and the coupling matrices. An example shows the proposed theoretical result is feasible and effective.
\end{abstract}

Keywords: pinning synchronization; reaction-diffusion neural networks; unknown time-varying coupling strengths; adaptive learning control; delay

\section{Introduction}

In the past few decades, neural networks (NNs) have become a hot topic due to their wide and important applications, such as signal transmission, image processing, machine learning, and pattern recognition, and so on. Most of the previous studies mainly concentrated on stability analysis, periodic or almost periodic attractors of NNs with or without delays [1-4]. However, it has been shown that delayed NNs can exhibit some complicated dynamics and even chaotic behaviors [5-11]. Based on the Lyapunov stability theory and Halanay inequality, by virtue of drive-response concept and time-delay feedback control techniques, reference [6] proposed several sufficient conditions for the exponential synchronization of two identical chaotic delayed NNs with stochastic perturbation. In reference [8], based on the linear matrix inequality (LMI) method, two delay-dependent criteria were derived to ensure the exponential stability of the error systems, which implied the master systems to synchronize with the slave systems. The non-fragile controller can be obtained by solving a set of LMIs. In reference [11], by introducing an improved Lyapunov-Krasovskii functional and employing convex combination approach, a delaydependent output feedback controller was derived to achieve synchronization with the help of a master-slave concept and linear matrix inequality approach.

Since chaos synchronization in an array of cellular NN systems by Wu and Chua [12] was investigated, the synchronization problems in arrays of coupled delayed NNs were investi-

○2014 Zhang et al.; licensee Springer. This is an Open Access article distributed under the terms of the Creative Commons Attribution License (http://creativecommons.org/licenses/by/2.0), which permits unrestricted use, distribution, and reproduction in any medium, provided the original work is properly cited. 
gated [13-17]. In reference [13], based on the Lyapunov function method and the specific property of the Householder transform, some criteria for the global synchronization of linearly coupled NN systems with time-varying coupling were obtained. Considering the effects of coupling delay, references $[14,15]$ addressed the global exponential synchronization in an array of coupled delayed NNs with both constant and delayed couplings. In practical applications, it was usually desirable to regulate a coupled network to a prescribed trajectory using external forces for the correct functioning of the whole network [18]. However, for a large-scale network, it is literally impossible to apply control actions to all nodes. Fortunately, many existing works have shown that one can adopt the pinning control strategy to synchronize a coupled network to a homogeneous trajectory, which means that one only needs to place local feedback injections on a small fraction of network nodes. Pinning control is an effective control scheme for controlling high-dimensional systems with numerous applications to turbulence, instabilities in plasma, multimode lasers, and reaction-diffusion systems, where the method could work in any region of parameter space and requires a significantly smaller number of controllers [19]. Moreover, for a large complex network, it is usually difficult to control it by adding controllers to all nodes. In order to reduce the number of controllers, a natural approach is to control the network by pinning parts of nodes. For a general reference on pinning control see references [20-22]. In the pinning control of coupled networks, it is well known that one of the most challenging problems is how to select a set of pinned nodes to guarantee the convergence of the pinning process. Reference [20] studied synchronization via pinning control on general complex dynamical networks, such as strongly connected networks, networks with a directed spanning tree, weakly connected networks, and directed forests. In reference [21], it was shown that the network can be pinned to a homogeneous state by applying adaptive feedback control actions to a small fraction of network nodes.

For many realistic networks, the node state in complex networks rely intensively on the time and space in many circumstances. As pointed out in reference [22], food webs attract increasing attention of researchers from different fields in recent years. A food web can be characterized by a model of complex network, in which a node represents a species. To our knowledge, species are usually inhomogeneously distributed in a bounded habitat and the different population densities of predators and preys may cause different population movements, so it is important to investigate their spatial density in order to better protect and control their population. In such a case, the state variable of node will represent the spatial density of the species. In addition, the whole structure and dynamic behavior of the multilayer cellular NNs are seriously dependent on the evolution time of each variable and its position (space), but also they are intensively dependent on its interactions deriving from the space-distributed structure of the whole networks [23]. Moreover, both in biological and man-made NNs when electrons are moving in asymmetric electromagnetic fields, strictly speaking, diffusion effects cannot be avoided, so we must consider that the activations vary in space as well as in time, which is expressed by partial differential equations [24-34]. [29] discussed the adaptive synchronization in an array of linearly coupled NNs with reaction-diffusion terms and time delays. In [31], the pinning control problem of linearly coupled RDNNs with unbounded time delays was also considered. The authors investigated adaptive synchronization phenomena for delayed RDNNs with unknown time-varying coupling strengths under stochastic perturbations [35]. 
Motivated by the work of $[29,31,32,35]$, in this paper we study the pinning synchronization in an array of linearly coupled delayed RDNNs with unknown time-varying coupling strengths and unknown time-varying delay, which is challenging and important both in theories and applications. How to incorporate adaptive pinning control techniques for time-delay systems into learning control to solve the synchronization problem of linearly coupled RDNNs with unknown time-varying parameters and unknown time-varying delays. As is well known, adaptive control can effectively deal with constant parametric uncertainties for systems. It is still an open problem how to control a system with unknown time-varying parameters. The main design difficulty is how to deal with the unknown time-varying parameters. Fortunately, this paper shows that this difficulty can be successfully overcome by constructing a Lyapunov-Krasovskii-like CEF. Suitable adaptive controllers are designed to ensure pinning synchronization for linearly coupled RDNNs. Compared with the previous works, the main contributions of this paper lie in the following. (1) We extend the existing concept of CEF to the time-delay setting, and a class of linearly coupled RDNNs with time-varying parameters and time-varying delays are given under the assumption that all time-varying parameters have a common periodicity. As far as we know, this extension has not been investigated in the literature at the present stage. (2) The CEF-based adaptive pinning control strategy, extended to delayed parabolic NNs. Thus, delayed parabolic NNs are further enlarged so that a CEF-based adaptive pinning control scheme can be designed for them. (3) From the viewpoint of technical innovation, we introduce a novel approach to handle unknown time-varying parameters in delayed parabolic NNs. We study the combination of learning control and adaptive pinning control leading to the asymptotic synchronization adaptive system in the presence of unknown time-varying parameters. In practice, the proposed synchronization controller will be easy to implement. Moreover, numerical simulations are provided to show the feasibility of the developed methods.

The paper is organized as follows. Section 2 provides some mathematical preliminaries and formulates the model of linearly coupled delayed RDNNs, some necessary definitions, lemmas, and hypotheses are presented. In Section 3, some criteria for the pinning synchronization of such linearly coupled RDNNs are derived. In Section 4, a numerical example is given to show the validity of the theoretical results. We conclude this paper in Section 5 .

Notation: Let $I_{N}$ be an $N$-dimensional identity matrix. For a symmetric matrix $M \in$ $R_{N \times N}$, denote the $i$ th row and the $i$ th column of $M$ as the $i$ th row-column pair and let $M_{l}$ represent a minor matrix of $M$ by removing arbitrary $l(1 \leq l \leq N)$ row-column pairs of $M$, denote $\lambda_{\min }(M)$ and $\lambda_{\max }(M)$ as the minimal and maximal eigenvalues of $M$, respectively. Let $C\left[(-\tau, 0] \times R^{m} ; R^{n}\right]$ denote the Banach space of continuous functions which map $(-\tau, 0] \times R^{m}$ into $R^{n}$ with the topology of uniform convergence. $\Omega=\left\{x|| x_{l} \mid<d_{l}, l=\right.$ $1,2, \ldots, m\}$ is a compact set with smooth boundary $\partial \Omega$ and mes $\Omega>0$ in space $R^{m}, d_{l}>0$ is a constant; $L^{2}(\Omega)$ is the space of real functions on $\Omega$ which are $L^{2}$ for the Lebesgue measure. It is a Banach space for the norm

$$
\|u(t, x)\|_{2}=\sqrt{\sum_{i=1}^{n}\left\|u_{i}(t, x)\right\|_{2}^{2}},
$$


where $u(t, x)=\left(u_{1}(t, x), \ldots, u_{n}(t, x)\right)^{T}$ and $\left\|u_{i}(t, x)\right\|_{2}=\left(\int_{\Omega}\left|u_{i}(t, x)\right|^{2} d x\right)^{1 / 2}$. In addition, we define

$$
\|u(t, x)\|_{\tau}=\sup _{-\tau \leq s \leq 0}\|u(t+s, x)\|_{2} .
$$

\section{Model description and preliminaries}

A single delayed RDNNs and Dirichlet boundary condition can be described by the following partial differential equations:

$$
\begin{aligned}
& \frac{\partial u_{i}(t, x)}{\partial t}= \sum_{l=1}^{m} \frac{\partial}{\partial x_{l}}\left(D_{i l} \frac{\partial u_{i}(t, x)}{\partial x_{l}}\right)-a_{i} u_{i}(t, x)+\sum_{j=1}^{n} w_{i j} g_{j}\left(u_{j}(t, x)\right) \\
&+\sum_{j=1}^{n} h_{i j} g_{j}\left(u_{j}(t-\tau(t), x)\right)+J_{i}, \quad t \geq 0, x \in \Omega, \\
& u_{i}(t, x)=0, \quad(t, x) \in(-\bar{\tau},+\infty) \times \partial \Omega, \\
& u_{i}(s, x)=\varphi_{i}(s, x), \quad(s, x) \in(-\bar{\tau}, 0] \times \Omega,
\end{aligned}
$$

where $x=\left(x_{1}, x_{2}, \ldots, x_{m}\right)^{T} \in \Omega, u_{i}(t, x)$ denotes the state the $i$ th neuron at time $t$ and in space $x ; a_{i}>0$ represents the rate with which the $i$ th unit will reset its potential to the resting state in isolation when disconnected from the networks and external inputs; $w_{i j}$ denotes the strength of the $j$ th unit on the $i$ th unit at time $t$ and in space $x ; h_{i j}$ is the synaptic connection strength of the $j$ th unit on the $i$ th unit at time $t$ and in space $x ; g_{j}\left(u_{j}(t, x)\right)$ denotes the activation function of the $j$ th unit at time $t$ and in space $x$ and $J=\left(J_{1}, J_{2}, \ldots, J_{n}\right)^{T}$ denotes a constant external input vector. $\tau(t)$ denotes the time-varying delay and satisfies $0 \leq \tau(t) \leq \bar{\tau}, 0 \leq \dot{\tau}(t) \leq \mu<1$, where $\bar{\tau}$ and $\mu$ are constants; $D_{l}=\operatorname{diag}\left(D_{1 l}, D_{2 l}, \ldots, D_{n l}\right)$ with $D_{i l} \geq 0$ stands for transmission diffusion operator along the $i$ th neuron. $\varphi_{i}(s, x)$ is a continuous and bounded function, $i, j=1,2, \ldots, n, l=1,2, \ldots, m$.

Let $\nabla \bullet Y=\left(\nabla \bullet Y_{1}, \nabla \bullet Y_{2}, \ldots, \nabla \bullet Y_{n}\right)^{T}, \nabla \bullet Y_{i}=\left(\frac{\partial y_{i 1}}{\partial x_{1}}, \frac{\partial y_{i 2}}{\partial x_{2}}, \ldots, \frac{\partial y_{i m}}{\partial x_{m}}\right)^{T}$, in which $Y=$ $\left[Y_{1}, Y_{2}, \ldots, Y_{n}\right]^{T}$ and $Y_{i}=\left(y_{i 1}, y_{i 2}, \ldots, y_{i m}\right)^{T}, i=1, \ldots, n$. For convenience, we rewrite system (1) as the following vector form:

$$
\begin{aligned}
& \frac{\partial u(t, x)}{\partial t}= \nabla \bullet(D \circ \nabla u(t, x))-A u(t, x)+W g(u(t, x)) \\
&+H g(u(t-\tau(t), x))+J, \quad t \geq 0, x \in \Omega, \\
& u(t, x)=0, \quad(t, x) \in(-\bar{\tau},+\infty) \times \partial \Omega, \\
& u(s, x)=\varphi(s, x), \quad(s, x) \in(-\bar{\tau}, 0] \times \Omega .
\end{aligned}
$$

Here

$$
\begin{aligned}
& A=\operatorname{diag}\left(a_{i}\right), \quad D=\left(D_{i l}\right)_{n \times m}, \quad W=\left(w_{i j}\right)_{n \times n}, \quad H=\left(h_{i j}\right)_{n \times n}, \\
& g(u(\cdot, x))=\left(g_{1}\left(u_{1}(\cdot, x)\right), g_{2}\left(u_{2}(\cdot, x)\right), \ldots, g_{n}\left(u_{n}(\cdot, x)\right)\right)^{T}, \\
& \nabla u(t, x)=\left(\nabla u_{1}(t, x), \nabla u_{2}(t, x), \ldots, \nabla u_{n}(t, x)\right)^{T}, \\
& \nabla u_{i}(t, x)=\left(\frac{\partial u_{i}(t, x)}{\partial x_{1}}, \frac{\partial u_{i}(t, x)}{\partial x_{2}}, \ldots, \frac{\partial u_{i}(t, x)}{\partial x_{n}}\right)^{T}, \quad i=1, \ldots, n,
\end{aligned}
$$

$D \circ \nabla u(t, x)=\left(D_{i l} \frac{\partial u_{i}(t, x)}{\partial x_{l}}\right)_{n \times m}$ is the Hadamard product of matrix $D$ and $\nabla u(t, x)$. 
In this paper, $N$ and such RDNNs (2) can be linearly coupled into a complex networks, which is characterized by

$$
\begin{aligned}
\frac{\partial z_{i}(t, x)}{\partial t}= & \nabla \bullet\left(D \circ \nabla z_{i}(t, x)\right)-A z_{i}(t, x)+W g\left(z_{i}(t, x)\right) \\
& +H g\left(z_{i}(t-\tau(t), x)\right)+J+c_{i}(t, x) \sum_{j=1}^{N} G_{i j} \Gamma z_{j}(t, x) \\
& +c_{i \tau}(t, x) \sum_{j=1}^{N} G_{\tau i j} \Gamma_{\tau} z_{j}(t-\tau(t), x), \quad i=1,2, \ldots, N,
\end{aligned}
$$

where $z_{i}(t, x)=\left(z_{i 1}(t, x), z_{i 2}(t, x), \ldots, z_{i n}(t, x)\right)^{T} \in R^{n}$ is the state vector of the $i$ th node, the matrices $\Gamma \in R^{n \times n}$ and $\Gamma_{\tau} \in R^{n \times n}$ describe inner-coupling of RDNNs at time $t$ and $t-\tau(t)$, respectively. $G=\left(G_{i j}\right)_{N \times N} \in R^{N \times N}$ and $G_{\tau}=\left(G_{\tau i j}\right)_{N \times N} \in R^{N \times N}$ are the coupling configuration and the delayed coupling configuration matrices, respectively. If there is a connection from node $i$ to the node $j(i \neq j)$ at time $t$, then the coupling $G_{i j}>0$, otherwise $G_{i j}=0(i \neq j)$. $G_{i i j}>0$ is defined similarly to $G_{i j}>0$, but it may not be identical to $G$. The diagonal elements of matrices $G$ and $G_{\tau}$ are defined as

$$
G_{i i}=-\sum_{j=1, i \neq j}^{N} G_{i j}, \quad G_{\tau i i}=-\sum_{j=1, i \neq j}^{N} G_{\tau i j}, \quad i=1,2, \ldots, N .
$$

The coupling matrices $G$ and $G_{\tau}$ are not required to be symmetric or irreducible. $c_{i}(t, x)$ and $c_{i \tau}(t, x)$ are unknown time-varying strengths for coupling and delayed coupling, respectively.

The boundary condition and the initial condition for system (3) are given in the following forms:

$$
\begin{aligned}
& z_{i j}(t, x)=0, \quad(t, x) \in(-\bar{\tau},+\infty) \times \partial \Omega, i=1,2, \ldots, N, j=1,2, \ldots, n, \\
& z_{i}(s, x)=\varphi_{i}(s, x), \quad(s, x) \in(-\bar{\tau}, 0] \times \Omega, i=1,2, \ldots, N,
\end{aligned}
$$

where $\varphi_{i}(s, x)=\left(\varphi_{i 1}(s, x), \varphi_{i 2}(s, x), \ldots, \varphi_{i n}(s, x)\right)^{T}$.

In order to obtain our main results, we assume the following conditions hold.

(A1) $g_{j}(\cdot)$ and $c_{i}^{*}(t, x), c_{i \tau}^{*}(t, x)$ satisfy the assumptions (A1) and (A2) in reference [35], respectively.

(A2) In the system (3), the inner coupling matrices $\Gamma, \Gamma_{\tau}$ and the diagonal elements of the matrix $G_{\tau}$ satisfy

$$
\|\Gamma\|=\gamma, \quad\left\|\Gamma_{\tau}\right\| \leq \gamma_{\tau}, \quad\left|G_{\tau i j}\right| \leq g_{\tau},
$$

where $\gamma, \gamma_{\tau}$ and $g_{\tau}$ are positive constants. Denote $\rho_{\min }$ as the minimum eigenvalue of the matrix $\left(\Gamma+\Gamma^{T}\right) / 2$.

(A3) Suppose $G_{i}$ is the minor matrix of $\left(\hat{G}^{T}+\hat{G}\right) / 2$ by removing the first $i-1$ rows and columns, where $\hat{G}$ is a modified matrix of $G$ via replacing the diagonal elements $G_{i i}$ by $\left(\rho_{\min } / \gamma\right) G_{i i}$, where $\rho_{\min }$ and $\gamma$ are denoted in (A2). 
Remark 1 Why do we consider periodically time-varying coupling strengths instead of more general ones? In fact, some works on the rejection or estimate of periodic disturbances (or parameters) has been widely reported, e.g., see [35-40], where the periodic disturbances are allowed to enter the controlled systems. As pointed out in reference [38], periodic disturbances often exist in many mechanical control systems such as industrial robots and numerical control machines or disturbances depending on the frequency of the power supply. Recently, there have, indeed, been publications on some physical systems that can be described by the model with periodic disturbances [40]. The above reasons motivate our work in this paper.

Remark 2 In [35], by constructing a differential-difference type learning law and an adaptive learning control law, based on the Lyapunov-Krasovskii-like CEF method, a novel sufficient condition was derived to ensure adaptive asymptotical synchronization in the mean square sense for stochastic delayed RDNNs with unknown time-varying coupling strengths. Why do we study the pinning synchronization of linearly coupled RDNNs with unknown time-varying coupling strengths and unknown time-varying delay in this paper? As we know now, real-world complex networks normally have a large number of nodes. So it is usually difficult to control a complex network by adding the controllers to all nodes. To reduce the number of the controllers, a natural approach is to control a complex network by pinning part of the nodes. Furthermore, we should point out here why the parameters $G_{i j}, G_{\tau i j}, c_{i}$ and $c_{i \tau}$ are necessary. In this paper, $G_{i j}$ denote the instantaneous coupling configuration, which play a key role in the synchronization process. $G_{\tau i j}$ denote the delayed coupling configuration for a lag (perhaps long term) effect, which exist in practice, and generally do not play a positive role in the synchronization process. $c_{i}$ and $c_{i \tau}$ denote the coupling strengths which can be adjusted out of the structure of the network. Thus, $c_{i}$ and $c_{i \tau}$ have important engineering and physical sense in the control and physical problem of synchronization.

Remark 3 The activation functions are typically assumed to be continuous, bounded, differentiable, and monotonically increasing, such as the functions of sigmoid type, and these conditions are no longer needed in this paper. Especially in some applications, one is required to use unbounded activation functions. For example, when NNs are designed for solving optimization problems in the presence of constraints (linear, quadratic, or more general programming problems), unbounded activations modeled by diode-like exponential-type functions are needed to impose constraints' satisfaction. The extension of the quoted results to the unbounded case is not straightforward. Different from the bounded case, where the existence of an equilibrium point is always guaranteed, for unbounded activations it may happen that there is no equilibrium point. When considering the widely employed piecewise-linear NNs, infinite intervals with zero slope are present in activations, and it is of great interest to drop the assumptions of strict increase and continuous first derivative for the activation. The absolute capacity of an associative memory model can be significantly improved by replacing the usual sigmoid activation functions with non-monotonic activation functions [41]. In this paper, the activation functions $g_{j}\left(u_{j}\right)$ also include some kinds of typical functions widely used in circuit design, such as nondifferentiable piecewise linear output functions of the form $g_{j}(y)=1 / 2(|y-1|-|y+1|)$ and non-monotonically increasing functions of the form of Gaussian and inverse Gaussian functions [42]. 
Lemma 1 ([43]) Let $A \in R^{n \times n}$ be symmetric. One has

$$
\lambda_{\min }(A) a^{T} a \leq a^{T} A a \leq \lambda_{\max }(A) a^{T} a, \quad \forall a \in R^{n} .
$$

Lemma 2 ([44]) For a symmetric matrix $M \in R^{N \times N}$ and a diagonal matrix $D=\operatorname{diag}\left(d_{1}, \ldots\right.$, $d_{l}, \underbrace{0, \ldots, 0}_{N-l})$ with $d_{i}>0, i=1, \ldots, l(1 \leq l \leq N)$, let $M-D=\left[\begin{array}{cc}A-\tilde{D} & B \\ B^{T} & M_{l}\end{array}\right], D=\operatorname{diag}\left(d_{1}, \ldots, d_{1}\right.$, $\underbrace{0, \ldots, 0}_{N-l})$ where $M_{l}$ is the minor matrix of $M$ by removing its first $l$ row-column pairs, $A$ and $B$ are matrices with appropriate dimensions, $\tilde{D}=\operatorname{diag}\left(d_{1}, \ldots, d_{l}\right)$. If $d_{i}>\lambda_{\max }\left(A-B M_{l}^{-1} B^{T}\right)$, $i=1, \ldots, l, M-D<0$ is equivalent to $M_{l}<0$.

The object is designing a controller $v_{i}(t, x)$ to achieve synchronization, we introduce adaptive pinning control strategy to nodes in the NNs (3). Then the controlled network is given by

$$
\begin{aligned}
\frac{\partial z_{i}(t, x)}{\partial t}= & \nabla \bullet\left(D \circ \nabla z_{i}(t, x)\right)-A z_{i}(t, x)+\operatorname{Wg}\left(z_{i}(t, x)\right) \\
& +H g\left(z_{i}(t-\tau(t), x)\right)+J+c_{i}(t, x) \sum_{j=1}^{N} G_{i j} \Gamma z_{j}(t, x) \\
& +c_{i \tau}(t, x) \sum_{j=1}^{N} G_{i j} \Gamma_{\tau} z_{j}(t-\tau(t), x)+v_{i}(t, x), \quad i=1,2, \ldots, N .
\end{aligned}
$$

Define the error vector by

$$
e_{i}(t)=z_{i}(t, x)-u(t, x), \quad i=1,2, \ldots, N
$$

The feedback control law is designed as follows:

$$
\begin{aligned}
v_{i}(t, x)= & -\frac{1}{2} p_{i}\left[\hat{c}_{i}^{*}(t, x)+\hat{c}_{i \tau}^{*}(t, x)+\hat{c}_{i}^{*}(t-\omega, x)+\hat{c}_{i \tau}^{*}(t-\omega, x)\right] e_{i}(t) \\
& -p_{i}\left[\hat{c}_{i}^{* *}(t, x)+\hat{c}_{i \tau}^{* *}(t, x)\right] e_{i}(t), \quad i=1, \ldots, l
\end{aligned}
$$

and $v_{i}(t, x)=0, i=l+1, \ldots, N$, where $v_{i}(t, x)=\left(v_{i 1}(t, x), v_{i 2}(t, x), \ldots, v_{i n}(t, x)\right)^{T}, p_{i}$ is a positive constant. $\hat{c}_{i}^{*}(t, x), \hat{c}_{i \tau}^{*}(t, x), \hat{c}_{i}^{* *}(t, x)$ and $\hat{c}_{i \tau}^{* *}(t, x)$ are estimations to $c_{i}^{*}(t, x), c_{i \tau}^{*}(t, x), c_{i}^{* *}$ and $c_{i \tau}^{* *}$, respectively. The time-varying periodic adaptive gains and the time-invariance update laws are designed as

$$
\begin{aligned}
& \hat{c}_{i}^{*}(t, x)= \begin{cases}\hat{c}_{i}^{*}(t-\omega, x)+\eta_{1 i}(t, x) e_{i}(t)^{T} e_{i}(t), & t \in[0,+\infty), \\
0, & t \in(-\omega, 0],\end{cases} \\
& \hat{c}_{i \tau}^{*}(t, x)= \begin{cases}\hat{c}_{i \tau}^{*}(t-\omega, x)+\eta_{2 i}(t, x) e_{i}(t)^{T} e_{i}(t), & t \in[0,+\infty), \\
0, & t \in(-\omega, 0]\end{cases}
\end{aligned}
$$

and

$$
\dot{\hat{c}}_{i}^{* *}(t, x)=-\theta_{1 i} e_{i}(t)^{T} e_{i}(t), \quad \dot{\hat{c}}_{i \tau}^{* *}(t, x)=-\theta_{2 i} e_{i}(t)^{T} e_{i}(t),
$$


respectively, in which

$$
\eta_{1 i}(t, x)=\left\{\begin{array}{ll}
0, & 0 \leq t<\frac{1}{3} \omega, \\
\bar{\eta}_{1 i}(t, x), & \frac{1}{3} \omega \leq t \leq \frac{2}{3} \omega, \\
1, & t>\frac{2}{3} \omega,
\end{array} \quad \eta_{2 i}(t, x)= \begin{cases}0, & 0 \leq t<\frac{1}{3} \omega \\
\bar{\eta}_{2 i}(t, x), & \frac{1}{3} \omega \leq t \leq \frac{2}{3} \omega \\
1, & t>\frac{2}{3} \omega,\end{cases}\right.
$$

one can choose $\bar{\eta}_{j i}(t, x), 0 \leq \bar{\eta}_{j i}(t, x) \leq 1, j=1,2$, such that $\eta_{1 i}(t, x)$ and $\eta_{2 i}(t, x)$ are increasing and continuous functions. And $\theta_{j i}, 0 \leq \theta_{j i} \leq 1, j=1,2$, are positive constants.

Definition 1 If there is a control input $v_{i}(t, x)$ such that

$$
\lim _{t \rightarrow \infty}\left\|z_{i}(t, x)-u(t, x)\right\|_{2}=0, \quad \text { for all } i=1,2, \ldots, N
$$

then the coupled NN (7) is said to be synchronized.

One gets the following error dynamical system:

$$
\begin{aligned}
\frac{\partial e_{i}(t)}{\partial t}= & \nabla \bullet\left(D \circ \nabla e_{i}(t)\right)-A e_{i}(t)+W f\left(e_{i}(t)\right) \\
& +H f\left(e_{i}(t-\tau(t))\right)+c_{i}(t, x) \sum_{j=1}^{N} G_{i j} \Gamma e_{j}(t) \\
& +c_{i \tau}(t, x) \sum_{j=1}^{N} G_{\tau i j} \Gamma_{\tau} e_{j}(t-\tau(t))+v_{i}(t, x), \quad i=1,2, \ldots, N,
\end{aligned}
$$

where $e_{i}(\cdot, x)=\left(e_{i 1}(\cdot, x), \ldots, e_{i n}(\cdot, x)\right)^{T}, f\left(e_{i}(\cdot, x)\right)=\left(g_{1}\left(z_{i 1}(\cdot, x)\right)-g_{1}\left(u_{1}(\cdot, x)\right), \ldots, g_{n}\left(z_{i n}(\cdot, x)\right)-\right.$ $\left.g_{n}\left(u_{n}(\cdot, x)\right)\right)^{T}$.

\section{Main results}

Theorem 1 Assume (A1)-(A3) hold. If there exist a natural number $1 \leq l \leq N$ and positive constants $q_{i}, L_{1}, L_{2}$ such that

$$
\lambda_{\max }\left(\left(\left(\hat{G}^{T}+\hat{G}\right) / 2\right)_{l}\right)<-\frac{\alpha}{c_{M} \gamma}
$$

holds, where $\alpha=\sum_{r=1}^{m}\left(-\frac{D_{r}^{*}}{d_{r}^{2}}\right)+\lambda_{\max }\left(\frac{1}{2} L^{2}\right)+\lambda_{\max }\left(-A+\frac{W^{T} W+H^{T} H}{2}\right)+\frac{N^{2} g^{2} \gamma_{\tau}^{2}}{4 \delta} c_{\tau M}+\frac{1}{1-\mu}\left(\lambda_{\max }\left(\frac{1}{2} \times\right.\right.$ $\left.\left.L^{2}\right)+\delta c_{\tau M}\right)$ and $D_{r}^{*}=\max _{1 \leq j \leq n}\left(D_{j r}\right)(1 \leq r \leq m)$ are positive constants, then the controlled coupled RDNN (7) is globally synchronized under the pinning adaptive controllers (8).

Proof Define the Lyapunov-Krasovskii composite energy functional as

$$
\begin{aligned}
V(t)= & \int_{\Omega}\left\{\frac{1}{2} \sum_{i=1}^{N} e_{i}^{T}(t) e_{i}(t)+\frac{1}{2} \sum_{i=1}^{l}\left[p_{i} \int_{t-\omega}^{t}\left(\tilde{c}_{i}^{*}(s, x)^{2}+\tilde{c}_{i \tau}^{*}(s, x)^{2}\right) d s\right]\right. \\
& +\frac{1}{2} \sum_{i=1}^{l} p_{i}\left[\theta_{1 i}^{-1}\left(\tilde{c}_{i}^{* *}(t, x)+L_{1}\right)^{2}+\theta_{2 i}^{-1}\left(\tilde{c}_{i \tau}^{* *}(t, x)+L_{2}\right)^{2}\right] \\
& \left.+\frac{\left(\lambda_{\max }\left(\frac{1}{2} L^{2}\right)+\delta c_{\tau M}\right)}{1-\mu} \sum_{i=1}^{N} \int_{t-\tau(t)}^{t} e_{i}^{T}(s) e_{i}(s) d s\right\} d x,
\end{aligned}
$$


where

$$
\begin{aligned}
& t \geq \omega, \quad P=\operatorname{diag}(p_{1}, \ldots, p_{l}, \underbrace{0, \ldots, 0}_{N-l}), \\
& c_{i}^{*}(t, x)=c_{i}^{*}(t-\omega, x), \quad c_{i \tau}^{*}(t, x)=c_{i \tau}^{*}(t-\omega, x), \\
& \tilde{c}_{i}^{*}(t, x)=c_{i}^{*}(t, x)-\hat{c}_{i}^{*}(t, x), \quad \tilde{c}_{i \tau}^{*}(t, x)=c_{i \tau}^{*}(t, x)-\hat{c}_{i \tau}^{*}(t, x), \\
& \tilde{c}_{i}^{* *}(t, x)=c_{i}^{* *}-\hat{c}_{i}^{* *}(t, x), \quad \tilde{c}_{i \tau}^{* *}(t, x)=c_{i \tau}^{* *}-\hat{c}_{i \tau}^{* *}(t, x) .
\end{aligned}
$$

Taking the derivative of (13), by (9), (10), and (A1) along the solutions of (11) yields

$$
\begin{aligned}
& \dot{V}(t, e(t)) \leq \int_{\Omega}\left\{\sum _ { i = 1 } ^ { N } e _ { i } ^ { T } ( t ) \left\{\nabla \bullet\left(D \circ \nabla e_{i}(t)\right)-A e_{i}(t)+W f\left(e_{i}(t)\right)\right.\right. \\
& +H f\left(e_{i}(t-\tau(t))\right)+c_{i}(t, x) \sum_{j=1}^{N} G_{i j} \Gamma e_{j}(t)+c_{i \tau}(t, x) \sum_{j=1}^{N} G_{\tau i j} \Gamma_{\tau} e_{j}(t-\tau(t)) \\
& -\frac{1}{2} p_{i}\left[\hat{c}_{i}^{*}(t, x)+\hat{c}_{i \tau}^{*}(t, x)+\hat{c}_{i}^{*}(t-\omega, x)+\hat{c}_{i \tau}^{*}(t-\omega, x)\right] e_{i}(t) \\
& \left.-p_{i}\left[\hat{c}_{i}^{* *}(t, x)+\hat{c}_{i \tau}^{* *}(t, x)\right] e_{i}(t)\right\} \\
& +\sum_{i=1}^{l} p_{i}\left\{\frac{1}{2}\left[\tilde{c}_{i}^{*}(t, x)^{2}+\tilde{c}_{i \tau}^{*}(t, x)^{2}\right]-\frac{1}{2}\left[\tilde{c}_{i}^{*}(t-\omega, x)^{2}+\tilde{c}_{i \tau}^{*}(t-\omega, x)^{2}\right]\right. \\
& \left.+\left[\theta_{1 i}^{-1}\left(\tilde{c}_{i}^{* *}(t, x)+L_{1}\right) \dot{\tilde{c}}_{i}^{* *}(t, x)+\theta_{2 i}^{-1}\left(\tilde{c}_{i \tau}^{* * *}(t, x)+L_{2}\right) \dot{\tilde{c}}_{i \tau}^{* *}(t, x)\right]\right\} \\
& +\sum_{i=1}^{N} \frac{1}{1-\mu}\left(\lambda_{\max }\left(\frac{1}{2} L^{2}\right)+\delta c_{\tau M}\right) \\
& \left.\times\left[e_{i}^{T}(t) e_{i}(t)-(1-\dot{\tau}(t)) e_{i}^{T}(t-\tau(t)) e_{i}(t-\tau(t))\right]\right\} d x \\
& =\int_{\Omega}\left\{\sum _ { i = 1 } ^ { N } e _ { i } ^ { T } ( t ) \left[\nabla \bullet\left(D \circ \nabla e_{i}(t)\right)-A e_{i}(t)+W f\left(e_{i}(t)\right)+H f\left(e_{i}(t-\tau(t))\right)\right.\right. \\
& \left.+\sum_{i=1}^{N} \sum_{j=1}^{N} c_{i}(t, x) e_{i}^{T}(t) G_{i j} \Gamma e_{j}(t)+\sum_{i=1}^{N} \sum_{j=1}^{N} c_{i \tau}(t, x) e_{i}^{T}(t) G_{\tau i j} \Gamma_{\tau} e_{j}(t-\tau(t))\right] \\
& +\sum_{i=1}^{l} e_{i}^{T}(t) p_{i}\left\{-\frac{1}{2}\left[\hat{c}_{i}^{*}(t, x)+\hat{c}_{i \tau}^{*}(t, x)+\hat{c}_{i}^{*}(t-\omega, x)+\hat{c}_{i \tau}^{*}(t-\omega, x)\right] e_{i}(t)\right. \\
& \left.-\left[\hat{c}_{i}^{* *}(t, x)+\hat{c}_{i \tau}^{* *}(t, x)\right] e_{i}(t)\right\} \\
& +\sum_{i=1}^{l} p_{i}\left\{\frac{1}{2}\left[\tilde{c}_{i}^{*}(t, x)^{2}+\tilde{c}_{i \tau}^{*}(t, x)^{2}\right]-\frac{1}{2}\left[\tilde{c}_{i}^{*}(t-\omega, x)^{2}+\tilde{c}_{i \tau}^{*}(t-\omega, x)^{2}\right]\right. \\
& \left.+\left[\theta_{1 i}^{-1}\left(\tilde{c}_{i}^{* *}(t, x)+L_{1}\right) \dot{\tilde{c}}_{i}^{* *}(t, x)+\theta_{2 i}^{-1}\left(\tilde{c}_{i \tau}^{* *}(t, x)+L_{2}\right) \dot{\tilde{c}}_{i \tau}^{* *}(t, x)\right]\right\}
\end{aligned}
$$




$$
\begin{aligned}
& +\sum_{i=1}^{N} \frac{1}{1-\mu}\left(\lambda_{\max }\left(\frac{1}{2} L^{2}\right)+\delta c_{\tau M}\right) \\
& \left.\times\left[e_{i}^{T}(t) e_{i}(t)-(1-\dot{\tau}(t)) e_{i}^{T}(t-\tau(t)) e_{i}(t-\tau(t))\right]\right\} d x .
\end{aligned}
$$

According to Green's formula, Lemma 1 in reference [35] and the Dirichlet boundary condition, one can obtain

$$
\begin{aligned}
\sum_{i=1}^{N} \int_{\Omega} e_{i}^{T}(t) \nabla \bullet\left(D \circ \nabla e_{i}(t)\right) d x & =-\sum_{i=1}^{N} \int_{\Omega} \sum_{j=1}^{n} \sum_{r=1}^{m} D_{j r}\left(\frac{\partial e_{i j}(t)}{\partial x_{r}}\right)^{2} d x \\
& \leq-\sum_{i=1}^{N} \int_{\Omega} \sum_{r=1}^{m} \frac{D_{r}^{*}}{d_{r}^{2}} e_{i}^{T}(t) e_{i}(t) d x
\end{aligned}
$$

In view of (A1), Lemma 1 , and $2 a^{T} b \leq a^{T} X a+b^{T} X^{-1} b, \forall a, b \in R^{n}$, we know that

$$
\begin{aligned}
\sum_{i=1}^{N} e_{i}^{T}(t)\left[-A e_{i}(t)+W f\left(e_{i}(t)\right)+H f\left(e_{i}(t-\tau(t))\right)\right] \\
\leq \sum_{i=1}^{N}\left[-e_{i}^{T}(t) A e_{i}(t)+\frac{1}{2} e_{i}^{T}(t) W^{T} W e_{i}(t)+\frac{1}{2} f^{T}\left(e_{i}(t)\right) f\left(e_{i}(t)\right)\right. \\
\left.\quad+\frac{1}{2} e_{i}^{T}(t) H^{T} H e_{i}(t)+\frac{1}{2} f^{T}\left(e_{i}(t-\tau(t))\right) f\left(e_{i}(t-\tau(t))\right)\right] \\
\leq \quad\left[\lambda_{\max }\left(-A+\frac{W^{T} W+H^{T} H}{2}\right)+\lambda_{\max }\left(\frac{1}{2} L^{2}\right)\right] e^{T}(t) e(t) \\
\quad+\lambda_{\max }\left(\frac{1}{2} L^{2}\right) e^{T}(t-\tau(t)) e(t-\tau(t)) .
\end{aligned}
$$

From $G_{\tau i j} \geq 0$ and (4), we have

$$
\left|G_{\tau i j}\right|<\left|G_{\tau i i}\right|
$$

By (A2), (17), and Lemma 2 in reference [35], we derive

$$
\begin{aligned}
& \sum_{i=1}^{N} \sum_{j=1}^{N} c_{i \tau}(t, x) e_{i}^{T}(t) G_{\tau i j} \Gamma_{\tau} e_{j}(t-\tau(t)) \\
& \quad \leq \frac{1}{2} c_{\tau M} \sum_{i=1}^{N} \sum_{j=1}^{N}\left(G_{\tau i j}\right)^{2} e_{i}^{T}(t) \Gamma_{\tau}^{T} Q \Gamma_{\tau} e_{i}(t)+\frac{1}{2} c_{\tau M} \sum_{i=1}^{N} \sum_{j=1}^{N} e_{j}^{T}(t-\tau(t)) Q^{-1} e_{j}(t-\tau(t)) \\
& \quad \leq \frac{N g^{2}}{2} c_{\tau M} \sum_{i=1}^{N} e_{i}^{T}(t) \Gamma_{\tau}^{T} Q \Gamma_{\tau} e_{i}(t)+\frac{N}{2} c_{\tau M} \sum_{j=1}^{N} e_{j}^{T}(t-\tau(t)) Q^{-1} e_{j}(t-\tau(t)) \\
& \quad=\frac{N^{2} g_{\tau}^{2} \gamma_{\tau}^{2}}{4 \delta} c_{\tau M} \sum_{i=1}^{N} e_{i}^{T}(t) e_{i}(t)+\delta c_{\tau M} \sum_{j=1}^{N} e_{j}^{T}(t-\tau(t)) e_{j}(t-\tau(t))
\end{aligned}
$$

where $Q=\frac{N}{2 \delta} I$. 
From (13)-(18) and (A3), we get

$$
\begin{aligned}
& \dot{V}(t, e(t)) \leq \int_{\Omega}\left\{\sum_{r=1}^{m}\left(-\frac{D_{r}^{*}}{d_{r}^{2}}\right) e^{T}(t) e(t)\right. \\
& +e^{T}(t)\left[\lambda_{\max }\left(-A+\frac{W^{T} W+H^{T} H}{2}\right)+\lambda_{\max }\left(\frac{1}{2} L^{2}\right)\right] e(t) \\
& +\lambda_{\max }\left(\frac{1}{2} L^{2}\right) e^{T}(t-\tau(t)) e^{T}(t-\tau(t)) \\
& +\frac{N^{2} g_{\tau}^{2} \gamma_{\tau}^{2}}{4 \delta} c_{\tau M} e^{T}(t) e(t)+\delta c_{\tau M} e^{T}(t-\tau(t)) e(t-\tau(t)) \\
& +c_{M} \sum_{i=1}^{N} \sum_{j=1, j \neq i}^{N} e_{i}^{T}(t) G_{i j} \Gamma e_{j}(t)+c_{M} \sum_{i=1}^{N} G_{i i} \rho_{\min } e_{i}^{T}(t) e_{i}(t) \\
& -\frac{1}{2} \sum_{i=1}^{l} p_{i} e_{i}^{T}(t)\left[\hat{c}_{i}^{*}(t, x)+\hat{c}_{i \tau}^{*}(t, x)+\hat{c}_{i}^{*}(t-\omega, x)+\hat{c}_{i \tau}^{*}(t-\omega, x)\right] e_{i}(t) \\
& -\sum_{i=1}^{l} p_{i} e_{i}^{T}(t)\left[\hat{c}_{i}^{* *}(t, x)+\hat{c}_{i \tau}^{* *}(t, x)\right] e_{i}(t) \\
& +\sum_{i=1}^{l} p_{i}\left\{\frac{1}{2}\left[\tilde{c}_{i}^{*}(t, x)^{2}+\tilde{c}_{i \tau}^{*}(t, x)^{2}\right]-\frac{1}{2}\left[\tilde{c}_{i}^{*}(t-\omega, x)^{2}+\tilde{c}_{i \tau}^{*}(t-\omega, x)^{2}\right]\right. \\
& \left.+\left[\theta_{1 i}^{-1}\left(\tilde{c}_{i}^{* *}(t, x)+L_{1}\right) \dot{\tilde{c}}_{i}^{* *}(t, x)+\theta_{2 i}^{-1}\left(\tilde{c}_{i \tau}^{* *}(t, x)+L_{2}\right) \dot{\tilde{c}}_{i \tau}^{* *}(t, x)\right]\right\} \\
& +\sum_{i=1}^{N} \frac{\left(\lambda_{\max }\left(\frac{1}{2} L^{2}\right)+\delta c_{\tau M}\right)}{1-\mu} \\
& \left.\times\left[e_{i}^{T}(t) e_{i}(t)-(1-\dot{\tau}(t)) e_{i}^{T}(t-\tau(t)) e_{i}(t-\tau(t))\right]\right\} d x \\
& \leq \int_{\Omega}\left\{e^{T}(t)(M-\bar{P}) e(t)\right\} d x
\end{aligned}
$$

where $M=\alpha I_{N}+c_{M} \gamma \frac{\hat{G}^{T}+\hat{G}}{2}, \bar{P}=\left(L_{1}+L_{2}+c_{m}+c_{\tau m}\right) P, \alpha=\sum_{r=1}^{m}\left(-\frac{D_{r}^{*}}{d_{r}^{2}}\right)+\lambda_{\max }(-A+$ $\left.\frac{W^{T} W+H^{T} H}{2}\right)+\frac{N^{2} g_{\tau}^{2} \gamma_{\tau}^{2}}{4 \delta} c_{\tau M}+\frac{1}{1-\mu}\left(\lambda_{\max }\left(\frac{1}{2} L^{2}\right)+\delta c_{\tau M}\right)+\lambda_{\max }\left(\frac{1}{2} L^{2}\right)$.

It is clear that matrix $M$ is symmetric. Let $M-\bar{P}=\left[\begin{array}{cc}\tilde{A}-\tilde{P} & \tilde{B} \\ \tilde{B}^{T} & M_{l}\end{array}\right]$, where $M_{l}$ is the minor matrix of $M$ by removing its first $l(1 \leq l \leq N)$ row-column pairs, $\tilde{A}$ and $\tilde{B}$ are matrices with appropriate dimensions, $\tilde{P}=\left(L_{1}+L_{2}+c_{m}+c_{\tau m}\right) \operatorname{diag}\left(p_{1}, \ldots, p_{l}\right)$. According to (12), (18), (19), and Lemma 2 , we derive $\lambda_{\max }\left(M_{l}\right) \leq \alpha+c_{M} \gamma \lambda_{\max }\left(\left(\left(\hat{G}^{T}+\hat{G}\right) / 2\right)_{l}\right)$, which implies that $M_{l}<0$. If we choose positive constants $L_{1}, L_{2}$, and $p_{i}$ such that $p_{i}^{*}>\lambda_{\max }\left(\tilde{A}-\tilde{B} M_{l}^{-1} \tilde{B}^{T}\right)$, $p_{i}^{*}=\left(L_{1}+L_{2}\right) p_{i}>0, i=1,2, \ldots, l$. It follows from Lemma 2 and $M_{l}<0$ that $M-D<0$. By (19), we get $\dot{V}(t, e(t)) \leq 0$ and $\dot{V}(t, e(t))=0$ if and only if $e(t)=0_{N n}$. Therefore, the set $\Xi=$ $\left\{e^{T}(t) \mid e(t)=0_{N n}\right\}$ is the largest invariant set contained in the set $\Xi=\left\{e^{T}(t) \mid \dot{V}(t, e(t))=\right.$ $0\}$ of system (11). According to the well-known LaSalle invariance principle [41], we can conclude $e(t) \rightarrow 0$ as $t \rightarrow+\infty$. That is, the pinning controlled network (7) asymptotically synchronizes to network (2) under the pinning adaptive controllers (8). This completes the proof. 
Remark 4 In [24-26, 28, 30], the synchronization for single delayed RDNN was discussed and a series of the sufficient conditions were given. Recently, adaptive synchronization to linearly coupled RDNNs was investigated in references [29,31], some sufficient conditions for adaptive synchronization of such a system were established. However, different from the above references, in this paper we develop pinning synchronization RDNNs with general coupling matrices and coupled unknown time-varying parameters. Furthermore, the condition for pinning synchronization is dependent on reaction-diffusion terms and the diffusion region $\Omega$.

Remark 5 Since we consider the RDNNs with unknown time-varying parameters, so it is more complicated. Based on learning control technique, the synchronization criterion is obtained by adjusting time-varying coupling strengths, which is different from the methods used in references $[29,31]$. The results of this paper are new and they complement previously known results.

Remark 6 It is difficult to get the entire information on the coupling matrix $G_{\tau}$, thus we apply the partial information that the diagonal elements of the coupling matrix strength for delayed configuration $G_{\tau i i}$ has the upper bound $g_{\tau}$. In this paper, the coupling matrix $G$ does not need to be symmetric. Compared with the results in paper [29, 31], to achieve pinning adaptive synchronization, we can adaptively tune the coupling strengths $c$ and $c_{\tau}$ in this paper. In addition, the controllers are independent of the information of the time delay, so it is very easy to implement.

Remark 7 For the pinning controlled network (7), one may choose periodic adaptive control. The adaptation laws (9) and (10) are difference-type and a differential-type updating laws, which deal with the unknown time-varying parameters and unknown timeinvariance parameters. This control method was originally proposed in reference [36]. Based on the learning control techniques in references [36-40], we study the pinning synchronization of linearly coupled delayed RDNNs with general coupling matrices. Indeed, it is quite challenging to design some appropriate pinning learning controllers.

Remark 8 Recently, many results have been obtained for complex dynamic networks with time-varying parameters [18]. However, the existing studies show that ordinary differential equations with the time-varying parameters were processed by the bounding technique. As far as we know, until now this extension has not been investigated in the existing literature on distributed parameter systems. From the viewpoint of the technical approach, the main design difficulty is how to deal with the unknown time-varying parameters and unknown time-varying delays. In this paper, the difficulty is successfully overcome via constructing a Lyapunov-Krasovskii-like CEF, applying inequality techniques and an adaptive pinning control method.

Remark 9 From the proof of Theorem 1, we can also derive similar results for the RDNNs with Neumann boundary conditions or mixed boundary conditions. Furthermore, we can easily see that the results and research method in this paper also can be extended to many other types of RDNNs such as the bidirectional associative memory NNs and CohenGrossberg NNs. 
Theorem 2 Suppose (A1)-(A3) hold. If there exist a natural number $1 \leq l \leq N$ and positive constants $q_{i}, L_{1}, L_{2}$ such that $\lambda_{\max }\left(\left(\left(\hat{G}^{T}+\hat{G}\right) / 2\right)_{l}\right)<-\frac{\tilde{\alpha}}{c_{M \gamma}}$ holds, where $\tilde{\alpha}=$ $\sum_{r=1}^{m}\left(-\frac{D_{r}^{*}}{d_{r}^{2}}\right)+\lambda_{\max }(-A)+\alpha^{*}+\frac{N^{2} g^{2} \gamma_{\tau}^{2}}{4 \delta} c_{\tau M}+\frac{\left(\beta^{*}+\delta c_{\tau M}\right)}{1-\mu}+\beta^{*}, \beta^{*}=\frac{1}{2} \max _{1 \leq j \leq n} \sum_{k=1}^{n} L^{2}\left|h_{k j}\right|$, $\alpha^{*}=\frac{1}{2} \max _{1 \leq j \leq n} \sum_{k=1}^{n}\left(\left|w_{j k}\right|+\left|h_{j k}\right|+L^{2}\left|w_{k j}\right|\right)$ are positive constants, then the system (7) is synchronized under the pinning adaptive controllers (8).

Proof The proof is quite similar to that of Theorem 1 . To avoid unnecessary duplications, here, we only provide a sketch of the proof and omit the details.

Consider the Lyapunov functional candidate:

$$
\begin{aligned}
V(t)= & \int_{\Omega}\left\{\frac{1}{2} \sum_{i=1}^{N} e_{i}^{T}(t) e_{i}(t)+\sum_{i=1}^{l}\left[e_{i}^{T}(t) e_{i}(t) \int_{t-\omega}^{t}\left(c^{*}(s, x)^{2}+c_{\tau}^{*}(s, x)^{2}\right) d s\right]\right. \\
& +\frac{1}{2} \sum_{i=1}^{l}\left[\theta_{1 i}^{-1}\left(c^{* *}(t, x)+L_{1}\right)^{2}+\theta_{2 i}^{-1}\left(c_{\tau}^{* *}(t, x)+L_{2}\right)^{2}\right] \\
& \left.+\frac{\left(\beta^{*}+\delta c_{\tau M}\right)}{1-\mu} \sum_{i=1}^{N} \int_{t-\tau(t)}^{t} e_{i}^{T}(s) e_{i}(s) d s\right\} d x .
\end{aligned}
$$

According to (A1) and Lemma 1, we get

$$
\begin{aligned}
\sum_{i=1}^{N} e_{i}^{T}(t) & {\left[-A e_{i}(t)+W f\left(e_{i}(t)\right)+H f\left(e_{i}(t-\tau(t))\right)\right] } \\
\leq & \sum_{i=1}^{N}\left[-e_{i}^{T}(t) A e_{i}(t)\right]+\frac{1}{2} \sum_{i=1}^{N}\left[\sum_{j=1}^{n} \sum_{k=1}^{n}\left(\left|w_{j k}\right|+\left|h_{j k}\right|+L^{2}\left|w_{k j}\right|\right) e_{i j}^{2}(t)\right] \\
+ & \frac{1}{2} \sum_{i=1}^{N} \sum_{j=1}^{n} \sum_{k=1}^{n} L^{2}\left|h_{k j}\right| e_{i j}^{2}(t-\tau(t)) \\
\leq & {\left[\lambda_{\max }(-A)+\alpha^{*}\right] e^{T}(t) e(t)+\beta^{*} e^{T}(t-\tau(t)) e(t-\tau(t)) . }
\end{aligned}
$$

The derivative of $V(t)$ along the trajectories of (11) gives

$$
\begin{aligned}
\dot{V}(t, e(t)) \leq & \int_{\Omega}\left\{\sum_{r=1}^{m}\left(-\frac{D_{r}^{*}}{d_{r}^{2}}\right) e^{T}(t) e(t)\right. \\
& +e^{T}(t)\left[\lambda_{\max }(-A)+\alpha^{*}\right] e(t)+\beta^{*} e^{T}(t-\tau(t)) e^{T}(t-\tau(t)) \\
& +\frac{N^{2} g^{2} \gamma_{\tau}^{2}}{4 \delta} c_{\tau M} e^{T}(t) e(t)+\delta c_{\tau M} e^{T}(t-\tau(t)) e^{T}(t-\tau(t)) \\
& +c_{M} \sum_{i=1}^{N} \sum_{j=1, j \neq i}^{N} e_{i}^{T}(t) G_{i j} \Gamma e_{j}(t)+c_{M} \sum_{i=1}^{N} G_{i i} \rho_{\min } e_{i}^{T}(t) e_{i}(t) \\
& -\frac{1}{2} \sum_{i=1}^{l} p_{i} e_{i}^{T}(t)\left[\hat{c}^{*}(t, x)+\hat{c}_{\tau}^{*}(t, x)+\hat{c}^{*}(t-\omega, x)+\hat{c}_{\tau}^{*}(t-\omega, x)\right] e_{i}(t) \\
& -\sum_{i=1}^{l} p_{i} e_{i}^{T}(t)\left[\hat{c}^{* *}(t, x)+\hat{c}_{\tau}^{* *}(t, x)\right] e_{i}(t)+\sum_{i=1}^{l} p_{i}\left[\left[\tilde{c}^{*}(t, x)^{2}+\tilde{c}_{\tau}^{*}(t, x)^{2}\right]\right. \\
& -\left[\tilde{c}^{*}(t-\omega, x)^{2}+\tilde{c}_{\tau}^{*}(t-\omega, x)^{2}\right]
\end{aligned}
$$




$$
\begin{aligned}
& \left.+\left[2 \theta_{1 i}^{-1}\left(\tilde{c}^{* *}(t, x)+L_{1}\right) \dot{\tilde{c}}^{* *}(t, x)+2 \theta_{2 i}^{-1}\left(\tilde{c}_{\tau}^{* *}(t, x)+L_{2}\right) \dot{\tilde{c}}_{\tau}^{* *}(t, x)\right]\right\} \\
& \left.+\sum_{i=1}^{N} \frac{\left(\beta^{*}+\delta c_{\tau M}\right)}{1-\mu}\left[e_{i}^{T}(t) e_{i}(t)-(1-\dot{\tau}(t)) e_{i}^{T}(t-\tau(t)) e_{i}(t-\tau(t))\right]\right\} d x \\
\leq & \int_{\Omega}\left\{e^{T}(t)\left[\tilde{\alpha} I_{N}+c_{M} \gamma \frac{\hat{G}^{T}+\hat{G}}{2}-\left(L_{1}+L_{2}+c_{m}+c_{\tau m}\right) P\right] e(t)\right\} d x .
\end{aligned}
$$

Analogously to the proof of Theorem 1, we can conclude that the pinning controlled network (7) asymptotically synchronizes to network (2) under the pinning adaptive controllers (8). This completes the proof.

Corollary 1 Suppose (A1)-(A3) hold. If there exist a natural number $1 \leq l \leq N$ and positive constants $q_{i}, L_{1}, L_{2}$ such that $\lambda_{l+1}<-\frac{\chi}{c_{M \gamma}}$ holds, where $\chi=\min \{\alpha, \tilde{\alpha}\}, \alpha$ and $\tilde{\alpha}$ are defined in Theorem 1 and Theorem 2, respectively. Then the delayed RDNN (7) is globally synchronized under the pinning adaptive controllers (8).

\section{An illustrative example}

A simple example is given in order to illustrate the usefulness of our main results. Our aim is to examine the adaptive synchronization characteristic of linearly coupled RDNNs with unknown time-varying coupling strengths.

Example 1 Consider the following 2-dimensional uncoupled delayed RDNNs:

$$
\begin{aligned}
& \frac{\partial u_{i}(t, x)}{\partial t}= D_{i 1} \frac{\partial^{2} u_{i}(t, x)}{\partial x^{2}}-a_{i} u_{i}(t, x)+\sum_{j=1}^{2} w_{i j} g_{j}\left(u_{j}(t, x)\right) \\
&+\sum_{j=1}^{2} h_{i j} g_{j}\left(u_{j}\left(t-\tau_{j}(t), x\right)\right), \quad t \geq 0, x \in \Omega, i=1,2, \\
& u_{i}(t, x)=0, \quad(t, x) \in(-\bar{\tau},+\infty) \times \partial \Omega, i=1,2, \\
& \varphi_{1}(s, x)=2 \sin (\pi x), \quad \varphi_{2}(s, x)=3 \sin (\pi x),
\end{aligned}
$$

where $x \in \Omega=\{x|| x \mid \leq 4\}, g_{j}(y)=\tanh (y)$ and $\tau_{j}(t)=\frac{e^{t}}{1+e^{t}}(j=1,2)$. It is easy to see that $g_{j}(\cdot)$ satisfies (A1), $L_{j}=1,0<\tau(t)<1$ and $\dot{\tau}_{j}(t)=\frac{e^{t}}{\left(1+e^{t}\right)^{2}} \leq \frac{1}{2}$.

Let $A=\left(\begin{array}{ll}1 & 0 \\ 0 & 1\end{array}\right), W=\left(\begin{array}{cc}2 & -0.1 \\ -5 & 3\end{array}\right), H=\left(\begin{array}{cc}-1.5 & -0.1 \\ -0.2 & -2.5\end{array}\right), D_{11}=D_{21}=0.1$. Then numerical simulation shows that system (22) has a chaotic attractor (see Figures 1-2). In the following, we design the pinning controllers for system (22).

Next, we present a dynamical system consisting of three linearly coupled RDNNs. The dynamical behavior of the entire array can be described by the following delayed RDNNs with unknown time-varying coupling strengths:

$$
\begin{aligned}
\frac{\partial z_{i}(t, x)}{\partial t}= & \nabla \bullet\left(D \circ \nabla z_{i}(t, x)\right)-A z_{i}(t, x)+W g\left(z_{i}(t, x)\right) \\
& +H g\left(z_{i}(t-\tau(t), x)\right)+c_{i}(t, x) \sum_{j=1}^{N} G_{i j} \Gamma z_{j}(t, x) \\
& +c_{i \tau}(t, x) \sum_{j=1}^{N} G_{\tau i j} \Gamma_{\tau} z_{j}(t-\tau(t), x)+v_{i}, \quad i=1,2,3,
\end{aligned}
$$




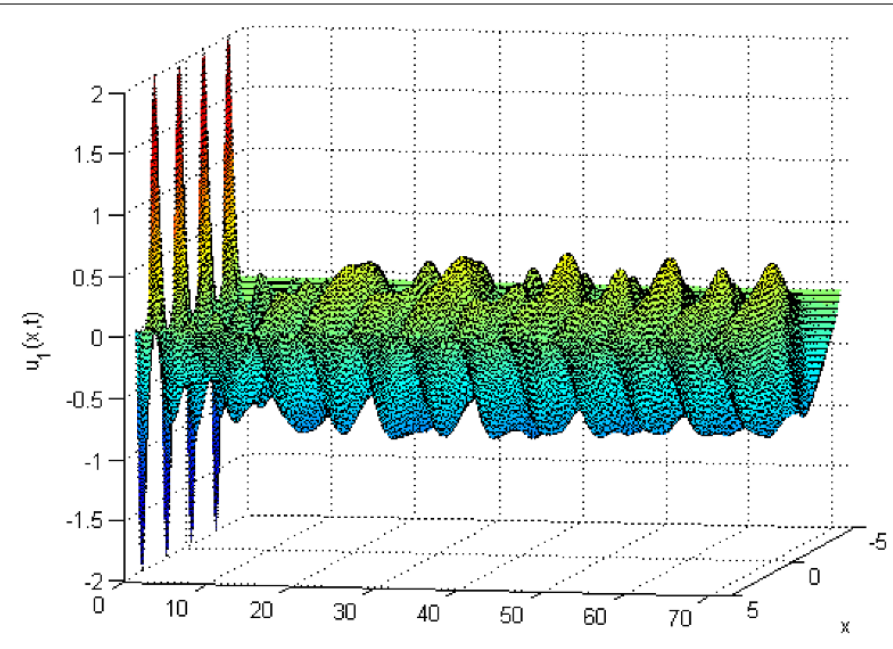

Figure 1 Chaotic behavior of the state $u_{1}(t, x)$ in system (20).

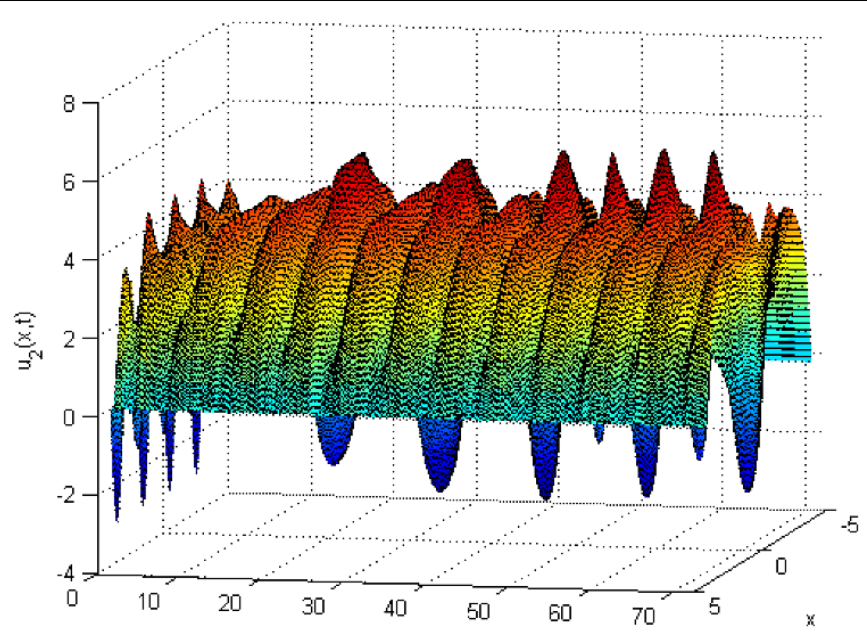

Figure 2 Chaotic behavior of the state $u_{2}(t, x)$ in system (20).

$$
\begin{aligned}
& z_{i}(t, x)=0, \quad(t, x) \in(-\bar{\tau},+\infty) \times \partial \Omega, i=1,2, \\
& z_{i 1}(s, x)=i \cos (\pi x), \quad z_{i 2}(s, x)=2 i \cos \left(\frac{1}{2} \pi x\right),
\end{aligned}
$$

where $z_{i}(t, x)=\left(z_{i 1}(t, x), z_{i 2}(t, x)\right)^{T}(i=1,2,3)$ is the state variable of the $i$ th NNs. The coupling configuration matrix is chosen to be

$$
G=\left(\begin{array}{ccc}
-2 & 1 & -1 \\
0 & -1 & 1 \\
2 & 1 & -3
\end{array}\right) \text {, }
$$

and the delayed coupling matrix is taken as $G_{\tau}=0.5 G$. The inner-coupling matrix is chosen as $\Gamma=\Gamma_{\tau}=I$. We choose the nodes 1 and 2 as pinned nodes. Choosing the adaptive 


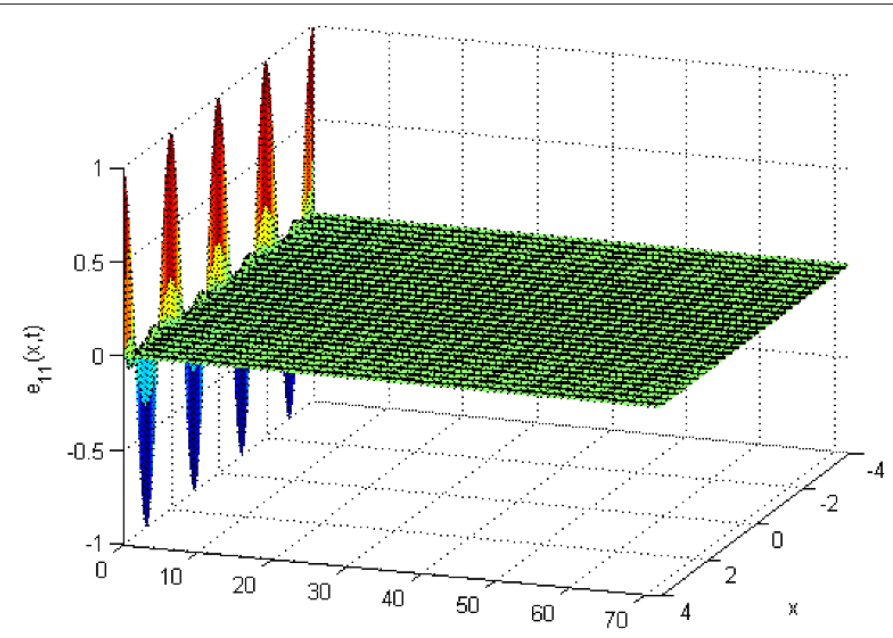

Figure 3 Asymptotical behavior of the error $e_{11}(t, x)$.

controllers we have

$$
\begin{aligned}
v_{i}(t, x)= & -\frac{1}{2} p_{i}\left[\hat{c}_{i}^{*}(t, x)+\hat{c}_{i \tau}^{*}(t, x)+\hat{c}_{i}^{*}(t-\omega, x)+\hat{c}_{i \tau}^{*}(t-\omega, x)\right] e_{i}(t) \\
& -p_{i}\left[\hat{c}_{i}^{* *}(t, x)+\hat{c}_{i \tau}^{* *}(t, x)\right] e_{i}(t), \quad i=1,2,
\end{aligned}
$$

and $v_{i}(t, x)=0, i=3$. The time-varying periodic adaptive gains and the time-invariance update laws are designed as (9) and (10).

Select the parameters of the above given system as follows:

$$
\begin{aligned}
& c_{1}^{*}(t, x)=12 \sin \frac{2 \pi t}{3}, \quad c_{2}^{*}(t, x)=10 \cos \pi t, \\
& c_{3}^{*}(t, x)=15 \sin \frac{2 \pi t}{3}, \quad c_{1 \tau}^{*}(t, x)=-2 \sin \frac{2 \pi t}{3}, \\
& c_{2 \tau}^{*}(t, x)=\cos \pi t, \quad c_{2 \tau}^{*}(t, x)=\cos \pi t, \\
& c_{1}^{* *}=118, \quad c_{2}^{* *}=110, \quad c_{3}^{* *}=120, \quad c_{1 \tau}^{* *}=3, \quad c_{2 \tau}^{* *}=2, \quad c_{3 \tau}^{* *}=1.5, \\
& \bar{\eta}_{1 i}(t, x)=0.5 t-1, \quad \bar{\eta}_{2 i}(t, x)=0.5 t-1, \quad p_{i}=0.3, \quad i=1,2 .
\end{aligned}
$$

The change processes of the state variables $u_{j}(t, x)$ and $e_{i j}(t)(i=1,2,3, j=1,2)$ are shown in Figures 1 to 8 . According to Theorem 1 and the simulation results, we can see that the coupled system (23) is synchronized.

\section{Conclusions}

In this paper, pinning synchronization of linearly coupled delayed RDNNs is discussed in detail. Some novel criteria for achieving network synchronization have been presented by utilizing some analytical and adaptive learning control techniques. It is interesting to find that a network can realize pinning synchronization via adaptively adjusting the unknown time-varying coupling strengths. The coupling configuration matrices are general, which are not required to be symmetric or irreducible. Moreover, some effective pinning schemes have been designed for networks with fixed structure and coupling strengths. 


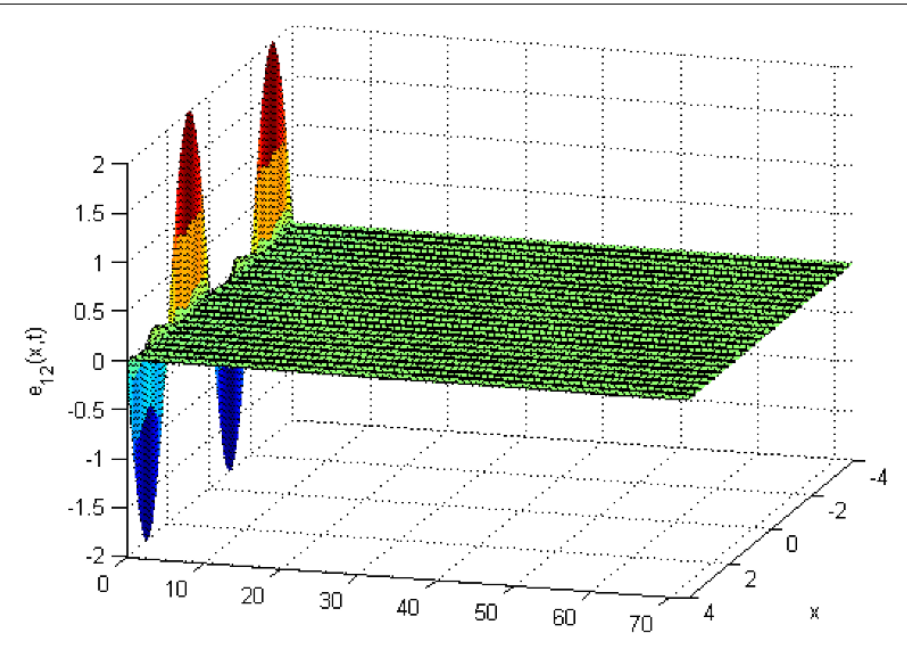

Figure 4 Asymptotical behavior of the error $e_{12}(t, x)$.

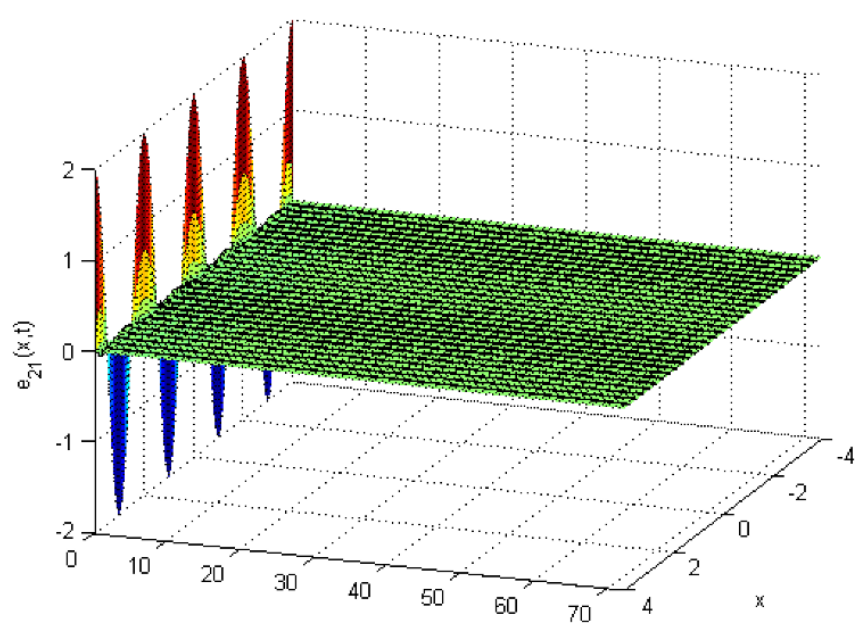

Figure 5 Asymptotical behavior of the error $e_{21}(t, x)$.

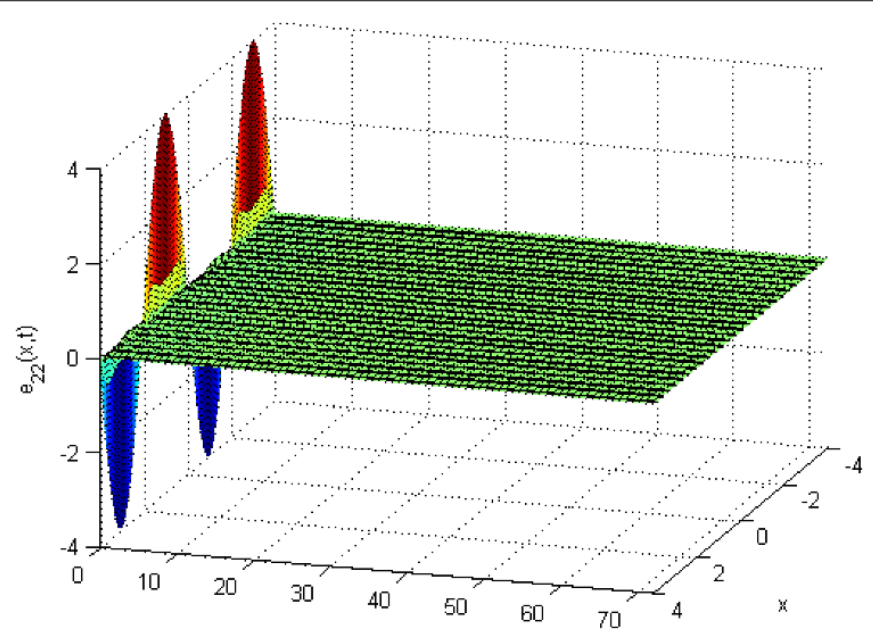

Figure 6 Asymptotical behavior of the error $e_{22}(t, x)$. 


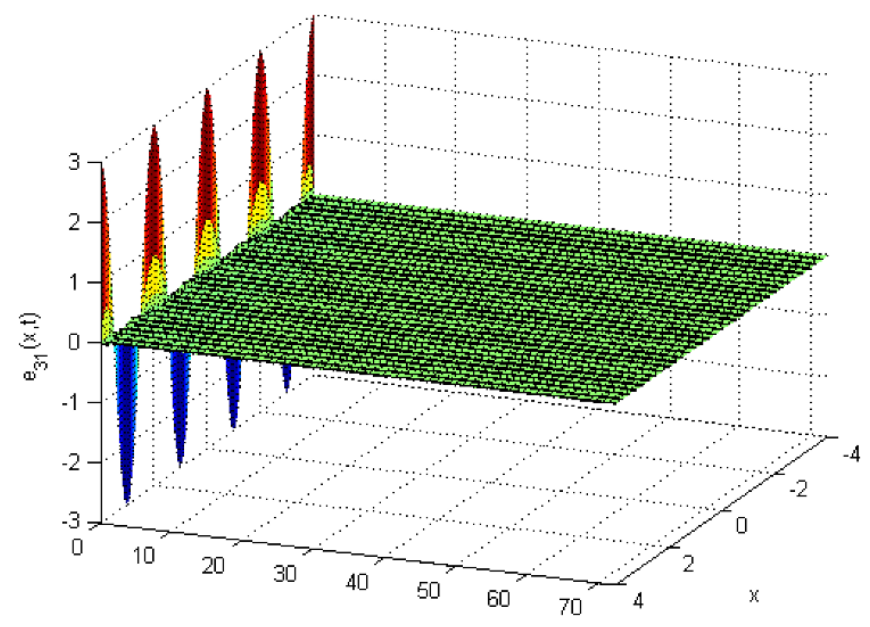

Figure 7 Asymptotical behavior of the error $e_{31}(t, x)$.

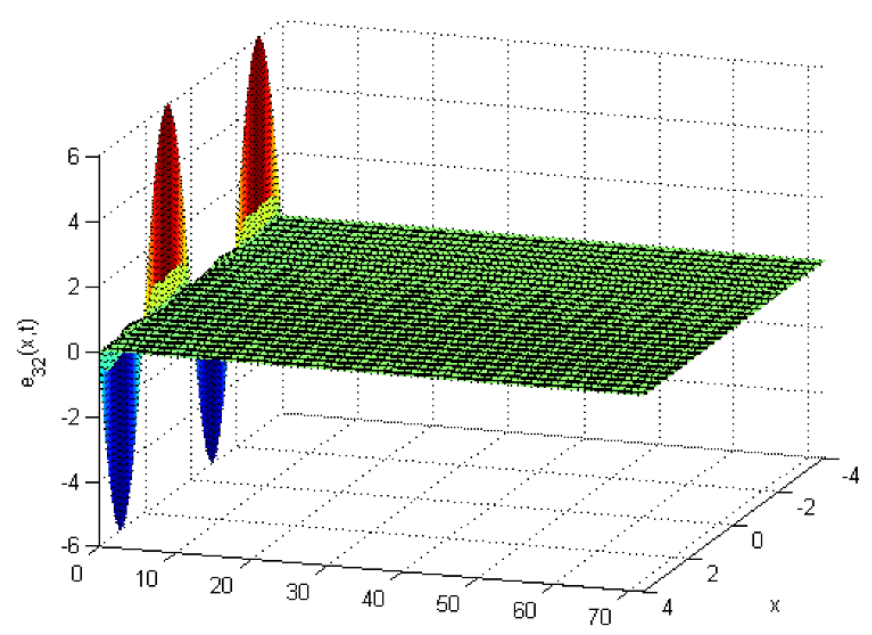

Figure 8 Asymptotical behavior of the error $e_{32}(t, x)$.

Compared with existing results, our synchronization is still very useful when the existing methods become invalid. Finally, the theoretical result is shown by a numerical example, which verifies well the theoretical analysis.

In fact, it is worth mentioning that there are still some important problems to solve for linearly coupled delayed RDNNs with unknown time-varying coupling strengths. From the literature, there are two common phenomena in many evolving networks: delay effects and impulsive effects. The states of electronic networks and biological networks are often subject to instantaneous disturbances and experience abrupt changes at certain instants, which may be caused by the switching phenomenon, frequency change, or me other sudden noise, i.e., they exhibit impulsive effects [45]. Impulsive dynamical networks, which are characterized by abrupt changes in the state differences of the systems at certain instants, have sparked the interest of many researchers for their various applications in information science, automated control systems, etc. Since delays and impulses can heavily affect the dynamical behaviors of NNs, it is necessary to investigate both delay and impul- 
sive effects on the synchronization of NNs. Very recently, the chaos synchronization of the Rikitake system based on Takagi-Sugeno fuzzy control techniques was studied [46]. The problem of sampled-data synchronization has been discussed for NNs with time-varying delay in the framework of the input delay approach [10]. To be more consistent with realistic networks, in the future, we will use these interesting control techniques to consider the pinning synchronization in a linearly coupled delayed RDNNs model with unknown time-varying strengths.

\section{Competing interests}

The authors declare that they have no competing interests.

\section{Authors' contributions}

WZ designed and performed all the steps of proof in this research and also wrote the paper. JL and MC participated in the design of the study and suggested many good ideas that made this paper possible and helped to draft the first manuscript. All authors read and approved the final manuscript.

\section{Author details}

${ }^{1}$ Institute of Mathematics and Applied Mathematics, Xianyang Normal University, Xianyang, 712000, P.R. China.

${ }^{2}$ Department of Mathematics, Xidian University, Xi'an, Shaanxi 710071, P.R. China.

\section{Acknowledgements}

This work is partially supported by the National Natural Science Foundation of China under Grant No. 60974139 and Project funded by China Postdoctoral Science Foundation under Grant No. 2013M540754, the Special research projects in Shaanxi Province Department of Education under Grant No. 2013JK0578 and Doctor Introduced project of Xianyang Normal University under Grant No. 12XSYK008.

Received: 27 December 2013 Accepted: 5 May 2014 Published: 14 May 2014

\section{References}

1. Arik, S: Stability analysis of delayed neural networks. IEEE Trans. Circuits Syst. I 47, 1089-1092 (2000)

2. Park, JH: Further note on global exponential stability of uncertain cellular neural networks with variable delays. Appl. Math. Comput. 188, 850-854 (2007)

3. Cao, J, Wang, J: Global asymptotic and robust stability of recurrent neural networks with time delays. IEEE Trans. Circuits Syst. I 52, 417-426 (2005)

4. Cao, J: New results concerning exponential stability and periodic solutions of delayed cellular neural networks. Phys. Lett. A 307, 136-147 (2003)

5. Park, JH: Synchronization of cellular neural networks of neutral type via dynamic feedback controller. Chaos Solitons Fractals 42, 1299-1304 (2009)

6. Sun, Y, Cao, J, Wang, Z: Exponential synchronization of stochastic perturbed chaotic delayed neural networks. Neurocomputing 70, 2477-2485 (2007)

7. Cheng, C, Liao, T, Yan, J, Wang, C: Exponential synchronization of a class of neural networks with time-varying delays. IEEE Trans. Syst. Man Cybern., Part B, Cybern. 36, 209-215 (2006)

8. Fang, M, Park, JH: Non-fragile synchronization of neural networks with time-varying delay and randomly occurring controller gain fluctuation. Appl. Math. Comput. 219(15), 8009-8017 (2013)

9. Jeong, SC, Ji, DH, Park, JH, Won, SC: Adaptive synchronization for uncertain chaotic neural networks with mixed time delays using fuzzy disturbance observer. Appl. Math. Comput. 219(11), 5984-5995 (2013)

10. Wu, Z-G, Park, JH, Su, H, Chu, J: Discontinuous Lyapunov functional approach to synchronization of time-delay neural networks using sampled-data. Nonlinear Dyn. 69(4), 2021-2030 (2012)

11. Balasubramaniam, $\mathrm{P}$, Vembarasan, $\mathrm{V}$ : Synchronization of recurrent neural networks with mixed time-delays via output coupling with delayed feedback. Nonlinear Dyn. 70, 677-691 (2012)

12. Wu, C, Chua, L: Synchronization in an array of linearly coupled dynamical system. IEEE Trans. Circuits Syst. I 42 , 430-447 (1995)

13. Wu, w, Chen, T: Global synchronization criteria of linearly coupled neural network systems with time-varying coupling. IEEE Trans. Neural Netw. 19, 319-332 (2008)

14. Cao, J, Li, P, Wang, W: Global synchronization in arrays of delayed neural networks with constant and delayed coupling. Phys. Lett. A 353, 318-325 (2006)

15. Chen, G, Zhou, J, Liu, Z: Global synchronization of coupled delayed neural networks and applications to chaotic CNN models. Int. J. Bifurc. Chaos 14, 2229-2240 (2004)

16. Lu, J, Ho, DWC, Cao, J, Kurths, J: Exponential synchronization of linearly coupled neural networks with impulsive disturbances. IEEE Trans. Neural Netw. 22, 329-336 (2011)

17. Park, MJ, Kwon, OM, Park, JH, Lee, SM, Cha, EJ: Synchronization criteria for coupled stochastic neural networks with time-varying delays and leakage delay. J. Franklin Inst. 349, 1699-1720 (2012)

18. Song, Q, Cao, J, Liu, F: Pinning synchronization of linearly coupled delayed neural networks. Math. Comput. Simul. 86 39-51 (2012)

19. Grigoriev, RO, Cross, MC, Schuster, HG: Pinning control of spatiotemporal chaos. Phys. Rev. Lett. 79(15), 2795-2798 (1997)

20. Yu, W, Chen, G, Lü, J, Kurths, J: Synchronization via pinning control on general complex networks. SIAM J. Control Optim. 51, 1395-1416 (2013) 
21. Yang, X, Cao, J, Lu, J: Synchronization of Markovian coupled neural networks with nonidentical node-delays and random coupling strengths. IEEE Trans. Neural Netw. Learn. Syst. 23, 60-71 (2012)

22. Wang, JL, Wu, HN, Guo, L: Novel adaptive strategies for synchronization of linearly coupled neural networks with reaction-diffusion terms. IEEE Trans. Neural Netw. Learn. Syst. 25, 429-440 (2014)

23. Chua, LO, Roska, T: The CNN paradigm. IEEE Trans. Circuits Syst. I, Fundam. Theory Appl. 40, 147-156 (1993)

24. Li, S, Yang, H, Lou, X: Adaptive exponential synchronization of delayed neural networks with reaction-diffusion terms. Chaos Solitons Fractals 40, 930-939 (2009)

25. Lou, X, Cui, B: Asymptotic synchronization of a class of neural networks with reaction-diffusion terms and time-varying delays. Comput. Math. Appl. 52, 897-904 (2006)

26. Zhang, W, Li, J: Global exponential stability of reaction-diffusion neural networks with discrete and distributed time-varying delays. Chin. Phys. B 20(3), 030701 (2011)

27. Wang, JL, Wu, HN, Guo, L: Passivity and stability analysis of reaction-diffusion neural networks with Dirichlet boundary conditions. IEEE Trans. Neural Netw. 22, 2105-2116 (2011)

28. Wang, Y, Cao, J: Synchronization of a class of delayed neural networks with reaction-diffusion terms. Phys. Lett. A 369 201-211 (2007)

29. Wang, K, Teng, Z, Jiang, H: Adaptive synchronization in an array of linearly coupled neural networks with reaction-diffusion terms and time delays. Commun. Nonlinear Sci. Numer. Simul. 17, 3866-3875 (2012)

30. Zhang, W, Li, J: Global exponential synchronization of delayed BAM neural networks with reaction-diffusion terms and the Neumann boundary conditions. Bound. Value Probl. 2012, 2 (2012). doi:10.1186/1687-2770-2012-2

31. Liu, X: Synchronization of linearly coupled neural networks with reaction-diffusion terms and unbound time delays. Neurocomputing 73, 2681-2688 (2010)

32. Li, J, Zhang, W, Chen, M: Synchronization of delayed reaction-diffusion neural networks via an adaptive learning control approach. Comput. Math. Appl. 65, 1775-1785 (2013)

33. Balasubramaniam, P, Vidhya, C: Global asymptotic stability of stochastic BAM neural networks with distributed delays and reaction-diffusion terms. J. Comput. Appl. Math. 234, 3458-3466 (2010)

34. Balasubramaniam, P, Vidhya, C: Exponential stability of stochastic reaction-diffusion uncertain fuzzy neural networks with mixed delays and Markovian jumping parameters. Expert Syst. Appl. 39, 3109-3115 (2012)

35. Zhang, W, Li, J, Chen, M: Adaptive synchronization of the stochastic delayed RDNNs with unknown time-varying parameters. Adv. Differ. Equ. 2013, 253 (2013). doi:10.1186/1687-1847-2013-253

36. Xu, JX, Tan, Y: A composite energy function based learning control approach for nonlinear systems with time-varying parametric uncertainties. IEEE Trans. Autom. Control 47, 1940-1945 (2002)

37. Chen, W, Jiao, L: Adaptive tracking for periodically time-varying and nonlinearly parameterized systems using multilayer neural networks. IEEE Trans. Neural Netw. 21, 345-351 (2010)

38. Tomizuka, M: Dealing with periodic disturbances in controls of mechanical systems. Annu. Rev. Control 32, 193-199 (2008)

39. Guo, X, Li, J: A new synchronization algorithm for delayed complex dynamical networks via adaptive control approach. Commun. Nonlinear Sci. Numer. Simul. 17, 4395-4403 (2012)

40. $\mathrm{Xu}, \mathrm{J}$ : A new periodic adaptive control approach for time-varying parameters with known periodicity. IEEE Trans. Autom. Control 49, 579-583 (2004)

41. Guo, S, Huang, L: Stability analysis of Cohen-Grossberg neural networks. IEEE Trans. Neural Netw. 17(1), 106-117 (2006)

42. Yuan, Z, Huang, L, Hu, D, Liu, B: Convergence of nonautonomous Cohen-Grossberg-type neural networks with variable delays. IEEE Trans. Neural Netw. 19(1), 140-147 (2008)

43. Horn, RA, Johnson, CR: Matrix Analysis. Cambridge University Press, Cambridge (1985)

44. Song, Q, Cao, J: On pinning synchronization of directed and undirected complex dynamical networks. IEEE Trans. Circuits Syst. I 57, 672-680 (2010)

45. Lu, J, Ho, DWC, Cao, J, Kurths, J: Exponential synchronization of linearly coupled neural networks with impulsive disturbances. IEEE Trans. Neural Netw. 22, 329-335 (2011)

46. Vembarasan, V, Balasubramaniam, P: Chaotic synchronization of Rikitake system based on T-S fuzzy control techniques. Nonlinear Dyn. 74, 31-44 (2013)

10.1186/1687-1847-2014-146

Cite this article as: Zhang et al.: Pinning adaptive synchronization analysis of linearly coupled delayed RDNNs with unknown time-varying coupling strengths. Advances in Difference Equations 2014, 2014:146

\section{Submit your manuscript to a SpringerOpen ${ }^{\ominus}$ journal and benefit from:}

- Convenient online submission

- Rigorous peer review

- Immediate publication on acceptance

- Open access: articles freely available online

- High visibility within the field

- Retaining the copyright to your article

Submit your next manuscript at $>$ springeropen.com 